\title{
Combining opioids and benzodiazepines: effects on mortality and severe adverse respiratory events
}

\author{
Martijn Boon ${ }^{1}$, Eveline van Dorp$^{1}$, Suzanne Broens ${ }^{2}$, Frank Overdyk ${ }^{3}$ \\ ${ }^{1}$ Department of Anesthesiology, Leiden university medical Center, Leiden, The Netherlands; ${ }^{2}$ Department of Anesthesiology, Netherlands cancer \\ institute, Amsterdam, The Netherlands; ${ }^{3}$ Department of Anesthesiology, Trident Health System, Charleston, SC, USA \\ Contributions: (I) Conception and design: All authors; (II) Administrative support: None; (III) Provision of study materials or patients: All authors; \\ (IV) Collection and assembly of data: All authors; (V) Data analysis and interpretation: All authors; (VI) Manuscript writing: All authors; (VII) Final \\ approval of manuscript: All authors. \\ Correspondence to: Dr. Martijn Boon, MD, PhD. Department of Anesthesiology, Leiden University Medical Center, H5-22, Albinusdreef 2, 2333 ZA \\ Leiden, The Netherlands. Email: m.boon@lumc.nl.
}

\begin{abstract}
Opioids and benzodiazepines are increasingly used alone or in combination. However, the combined use of these agents increases the risk for potentially lethal respiratory depression. This review summarizes current evidence on the effects of the combined use of opioids and benzodiazepines on mortality and severe respiratory adverse events. The results of 29 included manuscripts showed that concomitant use of opioids and benzodiazepines increased the risk for these outcomes in most of clinical and non-clinical settings. However, the risk for harm and benefit of the drug combination strongly correlates to its context and there are situations, such as in the hospice setting, where benefits may outweigh the risks.
\end{abstract}

Keywords: Opioids; benzodiazepines; mortality

Submitted Nov 29, 2019. Accepted for publication Dec 30, 2019.

doi: 10.21037/apm.2019.12.09

View this article at: http://dx.doi.org/10.21037/apm.2019.12.09

\section{Introduction}

Currently, the USA and many other western countries are experiencing not only increased use and abuse of opioids, but also of other drugs that affect central nervous system activity $(1,2)$. Particularly the use of benzodiazepines, the most commonly prescribed sedative, has seen a sharp increase over the last decades (3), and it is estimated that 3\% of the general population receives long-term prescriptions of these drugs (4). Benzodiazepines are approved for a variety of conditions, most notably anxiety and sleep disorders. In addition, they are generally considered to have a good overall safety profile. However, like opioids, benzodiazepines have a potential for dependence and intoxication during prolonged use and when used in high doses (5). Unbeknownst to many patients and prescribers, when benzodiazepines are prescribed in combination with opioids, there is a much greater danger of harm compared to either taken in isolation. Benzodiazepines and opioids both depress respiration, thereby increasing the risk for potentially lethal apnoea (6). Accumulating data indeed suggest that drugs like benzodiazepines, contribute significantly to opioid related fatalities (7). Having recognized this threat, a U.S. Centres for Disease Control and Prevention (CDC) 2016 guideline urged clinicians to avoid concurrent prescription of opioids and benzodiazepines whenever possible (8). In addition, the drug combination received a black box label by the U.S. Food and Drug Administration (FDA) highlighting the dangers of concomitant prescribing. Nevertheless, concurrent prescribing continues to be common practice among physicians (3). However, it is known that the risk for harm (or benefit) when using opioids or benzodiazepines is critically dependent on the context. For example, was the drug combination prescribed for treatment of anxiety and chronic pain or during palliative care, or was the harm from intentional or unintentional misuse or abuse? These differences affect the risk and incidence of severe adverse 
events. This review summarizes currently available evidence on the concomitant use of opioids and benzodiazepines on serious patient harm and categorizes the results according to various clinical and ambulatory settings.

\section{Methods}

The goal of this review was to evaluate and summarize current clinical evidence of the combined use of opioids and benzodiazepines on mortality and adverse respiratory outcomes. Secondary aims were to evaluate whether outcomes of such practice would differ among various clinical and non-clinical settings. To this end, a search query was composed using the following Mesh terms: "Analgesics, Opioid", "Hypnotics and Sedatives", "Benzodiazepine", "Mortality", "Apnoea", "Respiratory insufficiency" and "Heart arrest". With this query, PubMed data base was searched on 11 October 2019 without date range limits.

Title and abstracts were screened for inclusion in the review. Only original research manuscripts written in English language that investigated the interaction between opioids and benzodiazepines on major adverse events including death and (cardio-)pulmonary arrest were eligible. We chose to focus on manuscripts that primarily reported on the opioid-benzodiazepine interaction, as this interaction is the most common and clinically relevant interaction. Interaction of opioids and other classes of sedatives were not specifically searched but results of manuscripts that reported on these interactions are displayed for included manuscripts. Abstracts were independently screened by $M B$, FJO and ED; inconsistencies were resolved by consensus. Full text of eligible articles was obtained and included manuscripts were sorted based on the clinical setting: "abuse and addiction"; "palliative healthcare"; "inpatient healthcare"; "ambulatory healthcare".

\section{Pooled data analyses}

To quantify the risk for mortality, we performed analyses of combined data when available and if appropriate. These analyses were conducted using statistical package R (version 3.5.0) with the metafor package $(9,10)$. Data were analyzed using random effects models, assuming two sources of variance, within-study error and betweenstudy error. Separate analyses were performed for hazard ratios and incidence risk ratios. Heterogeneity was assessed by measuring the degree of inconsistency in the studies' results $\left(\mathrm{I}^{2}\right)$.

\section{Results}

The flow chart of the PubMed search is shown in Figure 1. The search yielded 1,862 unique manuscripts. Five potentially eligible manuscripts were identified through reference searching (i.e., snowball method). After careful screening of 1,867 titles and abstracts, full text of 133 papers were assessed for eligibility. The majority of the manuscripts studied only the effect of opioids or benzodiazepines alone and did not specifically investigate the interaction. These manuscripts were excluded. Finally, 29 manuscripts met the inclusion criteria and were included in this review (see Figure 1). The characteristics and main findings of these studies are presented in Tables 1-4. Thirteen manuscripts were assigned to the "abuse and addiction" category, one to "palliative healthcare" category, three to the "inpatient healthcare" category and twelve to the "ambulatory healthcare" category. The majority of studies were retrospective cohort studies, followed by post-mortem studies and 2 prospective studies. Outcomes reporting varied accordingly: most studies reported incidences or incidence risk ratios or odds- or hazard ratios for mortality, post-mortem studies reported proportional mortality or death ratios. Data of 10 retrospective studies originating from the "abuse and addiction" or "ambulatory healthcare" subcategories were suitable for the pooled analyses of hazard ratios (HR) and/or incidence risk ratio (IRR). Both analyses indicate that the risk for mortality is increased when opioids are concomitantly used with benzodiazepines [pooled HR $1.72(1.18-2.52) ; \mathrm{I}^{2}=97 \%$ and pooled IRR 2.51 (1.44-4.36); $\mathrm{I}^{2}=95 \%$; see Figure 2]. We will now discuss evidence for each category separately.

\section{Abuse and addiction}

In total, 13 manuscripts that reported on abuse or intentional misuse of opioids and sedatives, mostly benzodiazepines, were included in this review (see Table 1) (11-23). Besides the interaction between opioids and benzodiazepines, interactions between opioids and other centrally acting substances, such as cocaine, antidepressants and alcohol were also commonly reported $(12,13,15-18,20,23)$. The data came from post-mortem analyses of deceased subjects $(\mathrm{n}=3)(14,17,21)$, retrospective cohort studies $(n=9)(11-13,15,16,18-20,22)$, and one prospective study (23). All studied the risk of death or severe adverse events when using opioids, benzodiazepines or both. Post-mortem studies indicate that poly substance abuse 

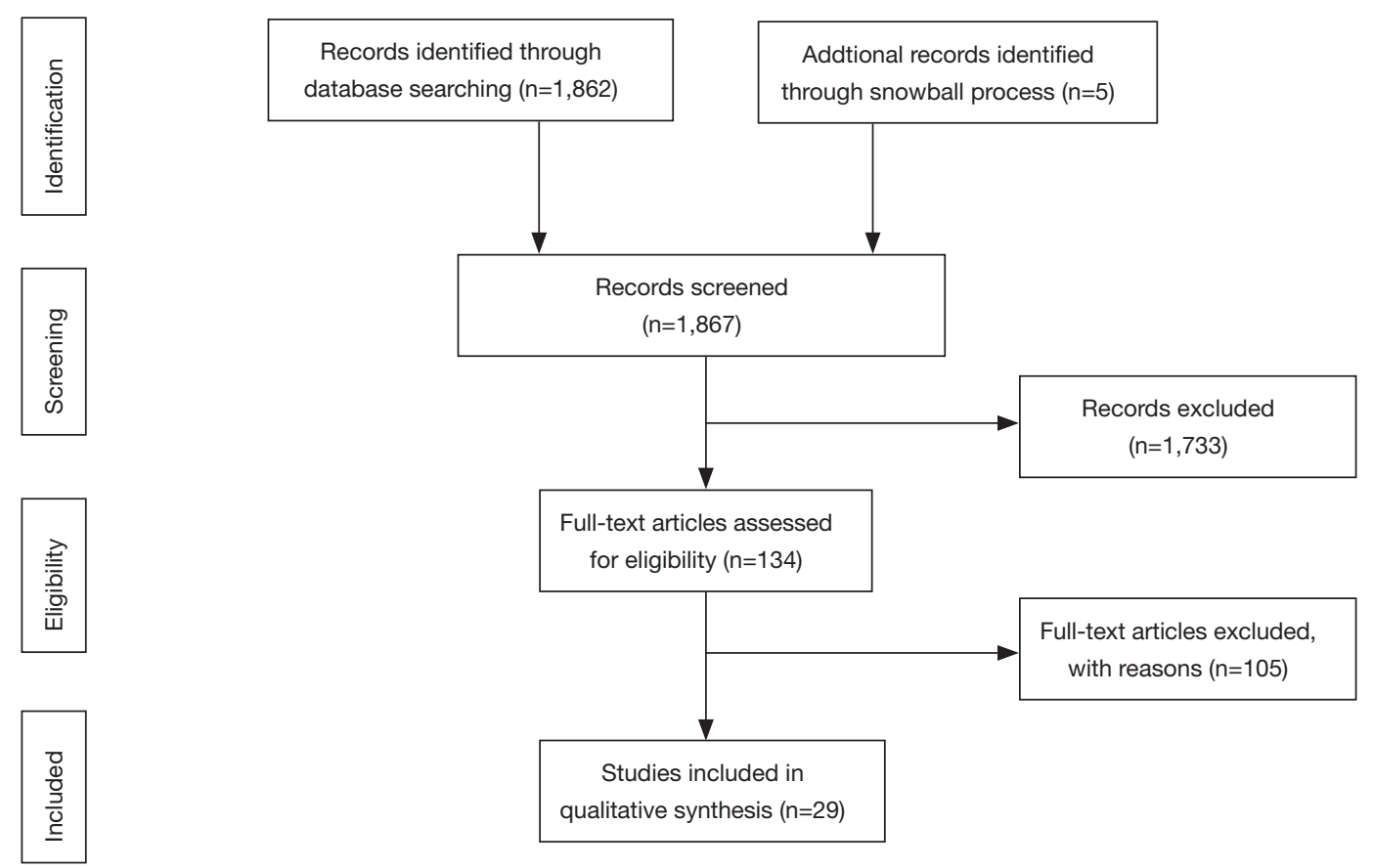

Figure 1 Consort flow-chart.

was common in deceased subjects, with incidences ranging from $20 \%$ to $80 \%(14,17,21)$. Overall, the majority of data show that the use of opioids with benzodiazepines or other centrally acting drugs has increased over the years, and that this drug combination increases the risk for mortality. Data of four studies were suitable for pooled analyses. These analyses also indicated a higher risk for mortality for combined use [pooled HR $1.36(1.07-1.72), \mathrm{I}^{2}=0 \%$; pooled IRR $1.77(0.32-9.75), \mathrm{I}^{2}=0 \%$; see part A in Figure 3 and part A in Figure 4]. The results of two studies diverge from the trending findings. Abrahamsson et al. did find a positive correlation between concomitant use with nonoverdose death, but not with overdose death and all-cause mortality (12). All-cause mortality however was significant in the unadjusted- and sensitivity analyses of this study. Second, Mirakbari et al. found no increased risk on adverse outcomes for intoxicated patients admitted to the emergency department (23). However, the methodology of this study was prone to inclusion and follow up bias In contrast, a study that reported on the treatment and outcomes after ICU admission for opioid intoxication found that patients who had also ingested benzodiazepines or amphetamines had a higher risk for mechanical ventilation and an increased length of stay (13).

A specific subgroup in this section consists of patients that are on opioid replacement therapy with buprenorphine or methadone $(12,18,19,22)$. Data from these studies indicate that benzodiazepines are involved in a significant part of fatalities in this subgroup. Interestingly, patients on methadone replacement therapy may be at higher risk for mortality and severe adverse respiratory events when concomitantly using benzodiazepines, than patients on buprenorphine replacement therapy $(12,19)$.

Finally, the manuscripts that reported on the effect of opioid combination with other CNS active substances, show an increased the risk for mortality, albeit the risks differed substantially between manuscripts and drug combination $(12,13,15-18,20,23)$.

In conclusion, concomitant use of benzodiazepines or other CNS active drugs by active opioid abusers and those on opioid replacement therapy substantially increases the risk for mortality.

\section{Palliative healthcare}

One manuscript was identified that reported on the use of opioids combined with benzodiazepines or antipsychotics such as haloperidol in terminally ill patients admitted to a hospice service (24). This study found that survival in terminally ill patients was not reduced by concomitant use 


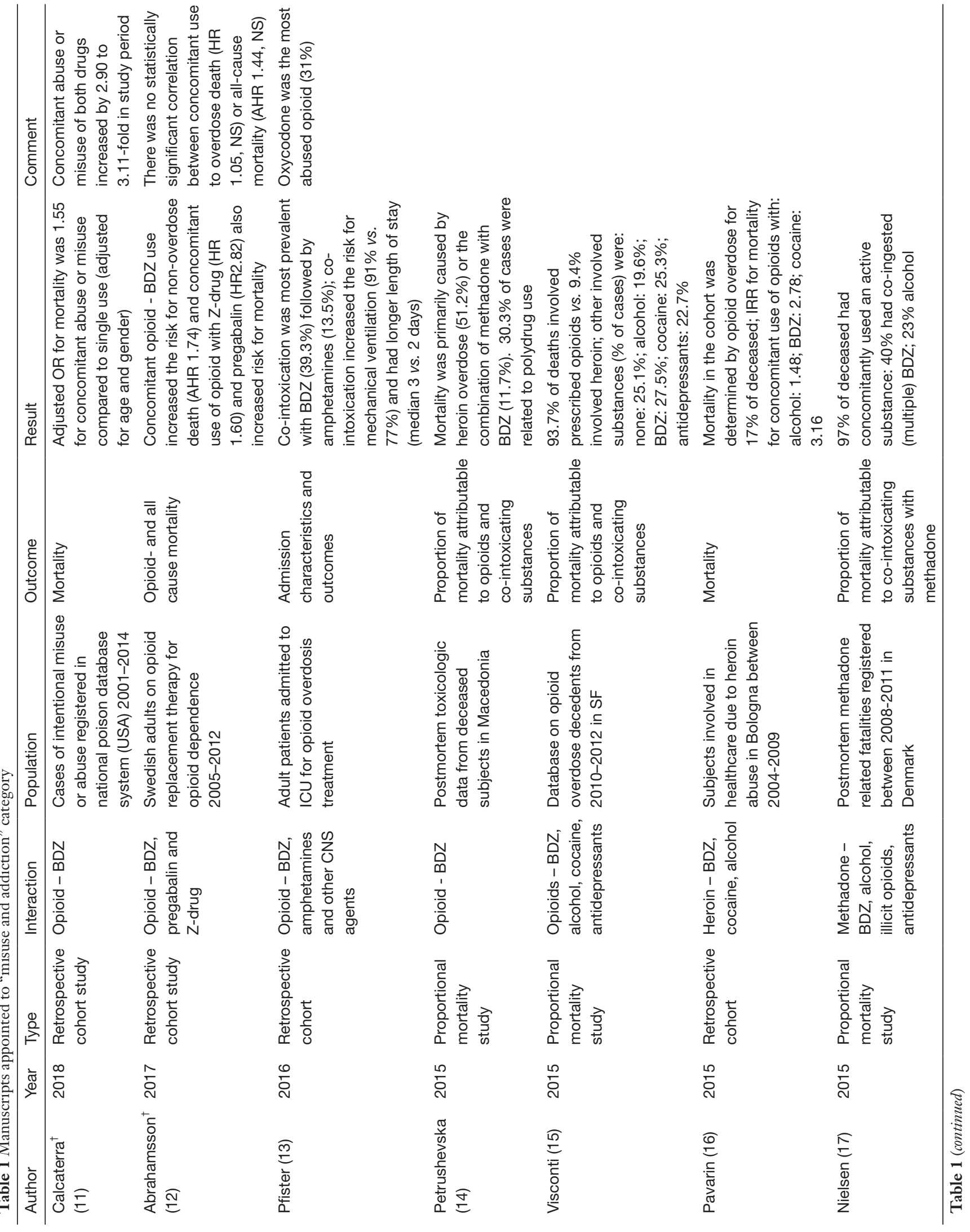




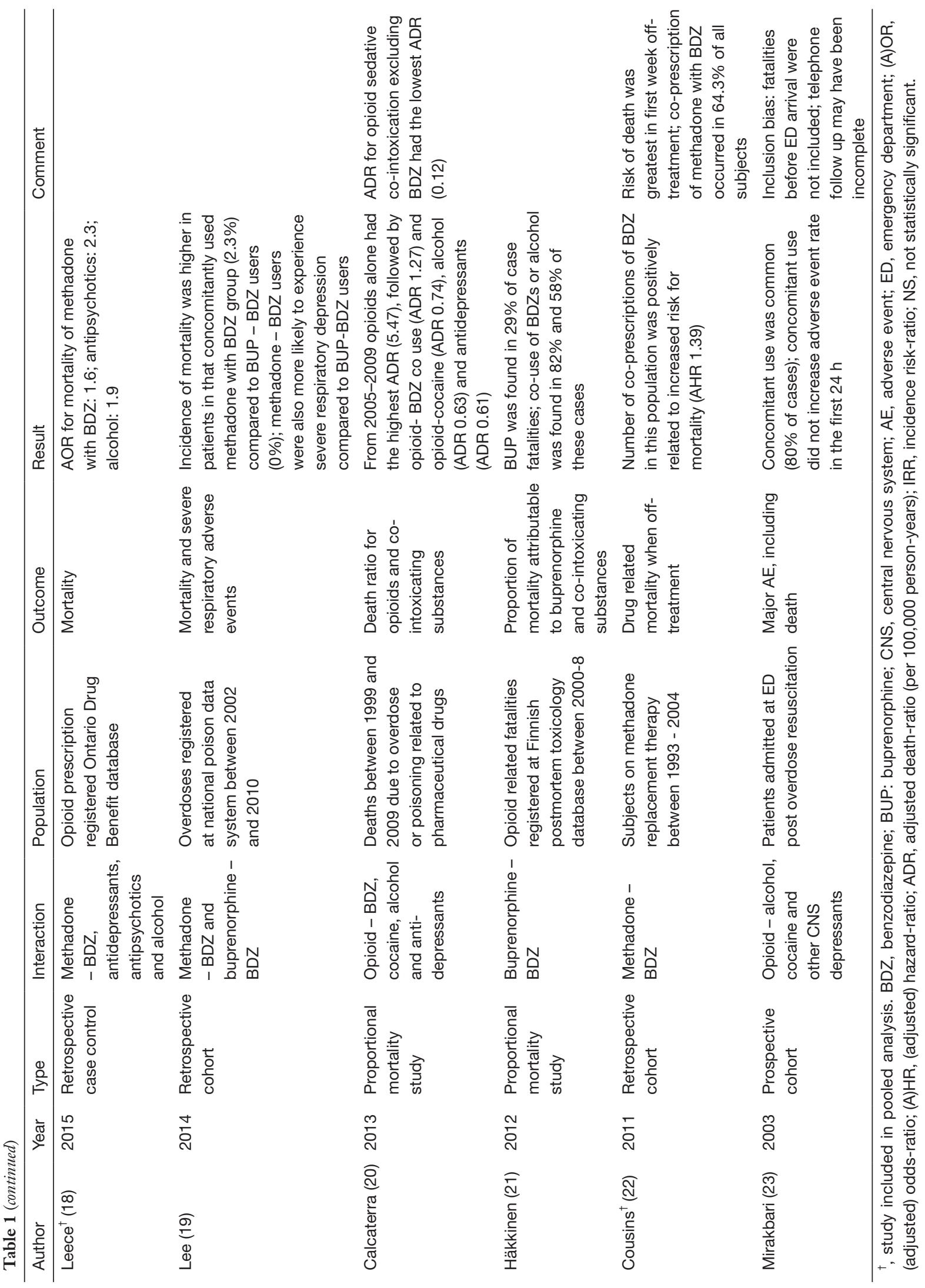


Table 2 Manuscript appointed to "palliative healthcare" category

\begin{tabular}{|c|c|c|c|c|c|c|c|}
\hline Author & Year & Type & Interaction & Population/setting & Outcome & Result & Comment \\
\hline
\end{tabular}

BDZ, benzodiazepine; HR, hazard-ratio; OME, oral morphine equivalents; NS, not statistically significant.

Table 3 Manuscripts appointed to "inpatient healthcare" category

\begin{tabular}{|c|c|c|c|c|c|c|c|}
\hline Author & Year & Type & Interaction & Population & Outcome & Result & Comment \\
\hline Izrailtyan (26) & 2018 & $\begin{array}{l}\text { Retrospective } \\
\text { cohort study }\end{array}$ & $\begin{array}{l}\text { Opioid - } \\
\text { sedative }\end{array}$ & $\begin{array}{l}>18 \text { years; in } \\
\text { hospital CPRA, RA } \\
\text { or CPR in surgical } \\
\text { and non-surgical } \\
\text { patients }\end{array}$ & Risk for CPRA & $\begin{array}{l}\text { Opioids and sedatives } \\
\text { independently and } \\
\text { additively increased risk } \\
\text { for CPRA; AOR for opioid } \\
\text { BDZ in medical patients } \\
3.83 \text { and } 2.34 \text { in surgical } \\
\text { patients }\end{array}$ & $\begin{array}{l}\text { Hispanic origin, } \\
\text { surgery for } \\
\text { malignancy, liver } \\
\text { disease, obesity } \\
\text { and COPD further } \\
\text { increased the risk } \\
\text { for CPRA }\end{array}$ \\
\hline Overdyk (27) & 2016 & $\begin{array}{l}\text { Retrospective } \\
\text { cohort study }\end{array}$ & $\begin{array}{l}\text { Opioid - } \\
\text { sedative }\end{array}$ & $\begin{array}{l}\text { Adult inpatient } \\
\text { discharges } \\
\text { reported in } \\
\text { premier database }\end{array}$ & $\begin{array}{l}\text { Risk and } \\
\text { outcome of in } \\
\text { hospital CPRA }\end{array}$ & $\begin{array}{l}\text { Opioids and sedatives } \\
\text { independently (AOR 1.81) } \\
\text { and additively (AOR 3.47) } \\
\text { increase the risk for in } \\
\text { hospital CPRA }\end{array}$ & \\
\hline
\end{tabular}

BDZ, benzodiazepine; CPRA, cardiopulmonary and respiratory arrest; (A)OR, (adjusted) odds-ratio; (A)HR, (adjusted) hazard-ratio; ADR, adjusted death-ratio (per 100,000 person-years); IRR, incidence risk-ratio; NS, not statistically significant.

of an opioid with a benzodiazepine or antipsychotic. In fact, the chance of surviving longer in this setting was higher for patients that used all three classes of medication. In addition, night-time death percentage was not increased in patients taking a drug combination. Finally, it was found that patients receiving opioid doses of more than $300 \mathrm{mg}$ oral morphine equivalents survived longer compared to patients on receiving lower doses. In conclusion, data from one study suggests that concomitant use of opioids with benzodiazepines or antipsychotics in the hospice setting may be safe. Additional research is needed to corroborate these results.

\section{Inpatient healthcare}

The search yielded three manuscripts that reported on the effect of opioid and sedative use in hospitalized patients (25-27). The studies by Overdyk et al. and Izrailtyan et al. 


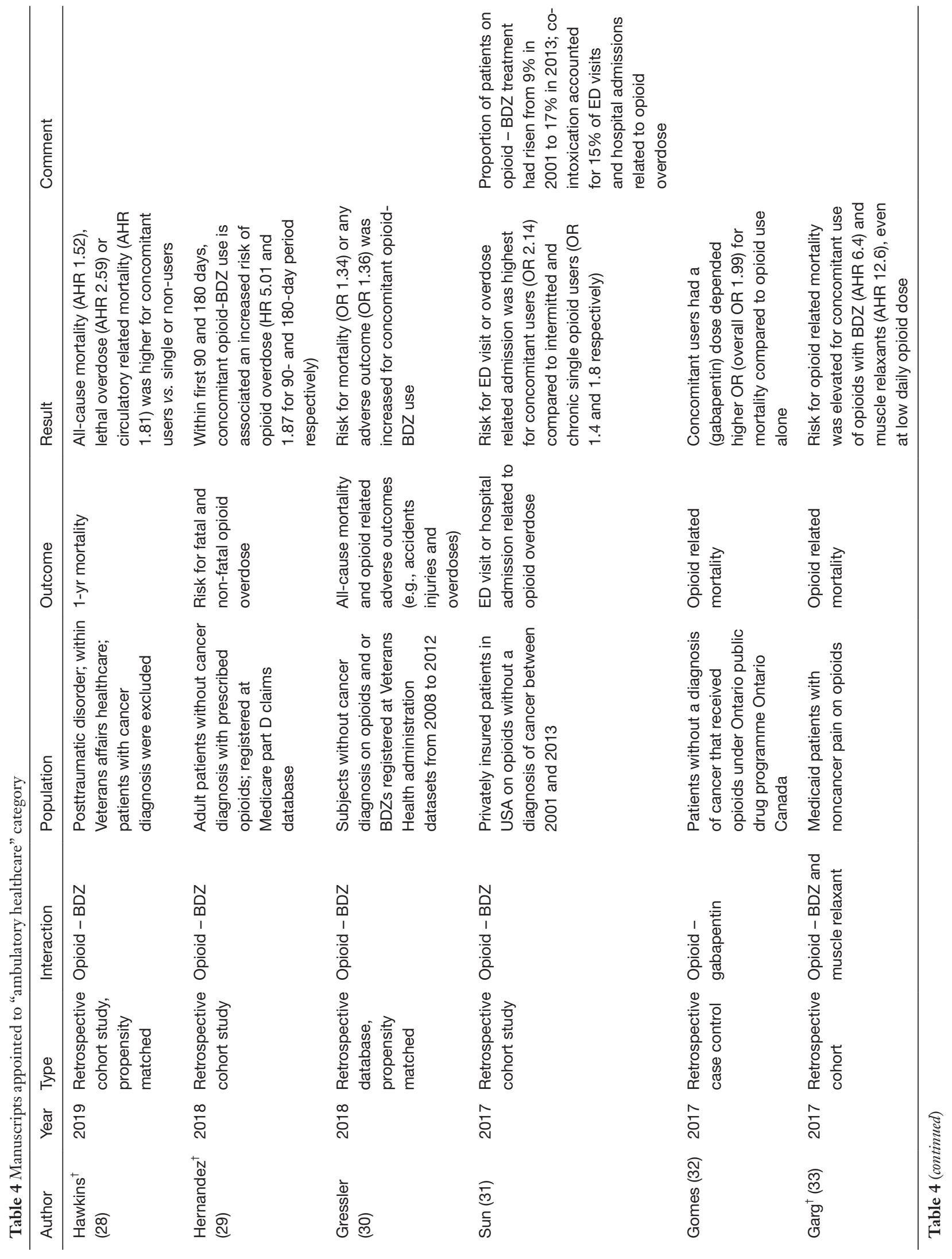




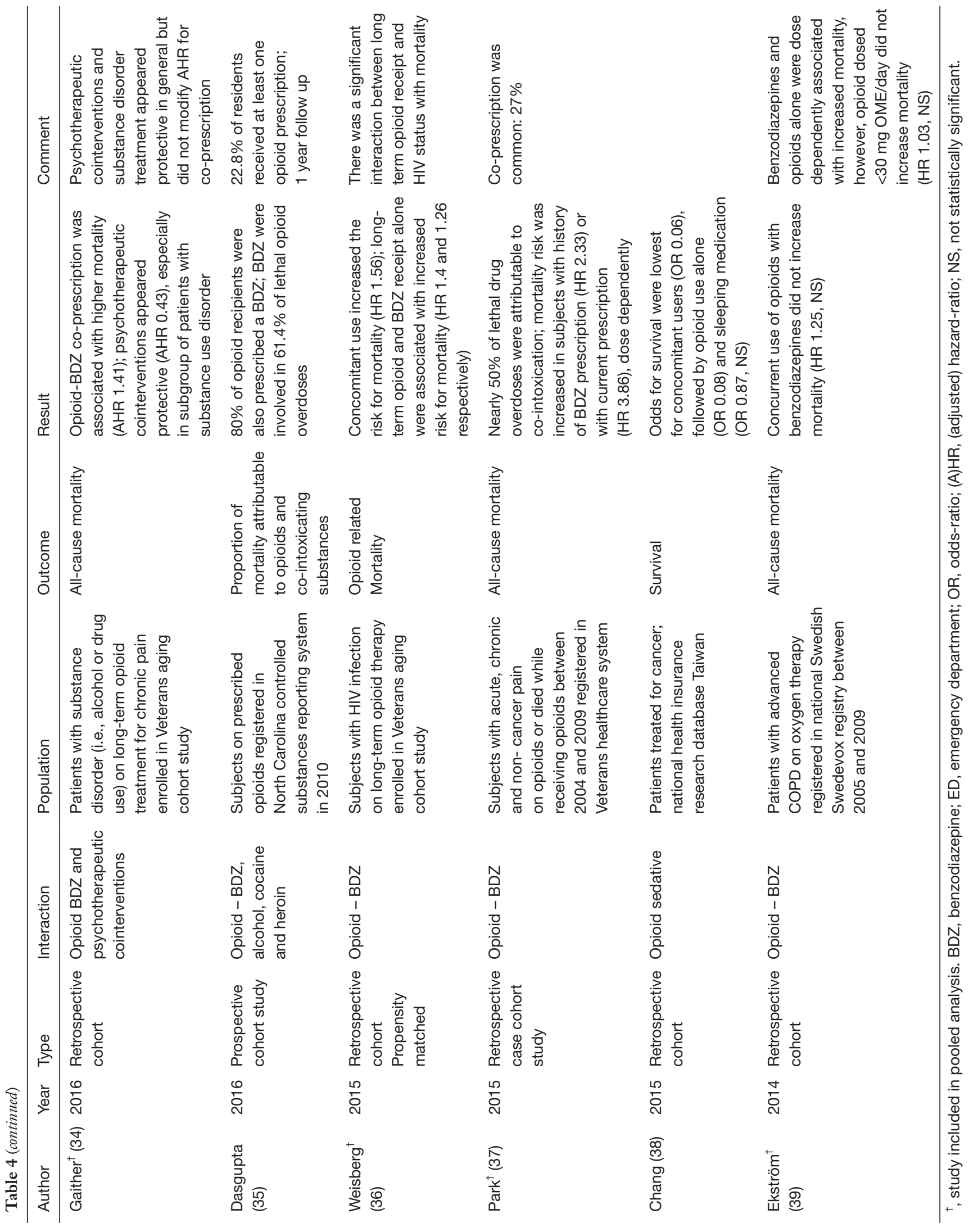


A

$\begin{array}{lrr}\text { Study } & \ln (\mathbf{H R}) \ln (\mathbf{s e H R}) \\ & 0.05 & 0.3670 \\ \text { Abrahamsson } & 0.29 & 0.0723 \\ \text { Cousins } & 0.49 & 0.0671 \\ \text { Gaither } & 0.42 & 0.1472 \\ \text { Hawkins } & 0.49 & 0.2076 \\ \text { Leece } & 1.35 & 0.0509 \\ \text { Park } & 0.43 & 0.0987 \\ \text { Weisberg } & \\ \text { Random effects model } & \\ \text { Prediction interval } & \\ \text { Heterogeneity: } I^{2}=97 \%, \tau^{2}=0.1296, p<0.01\end{array}$

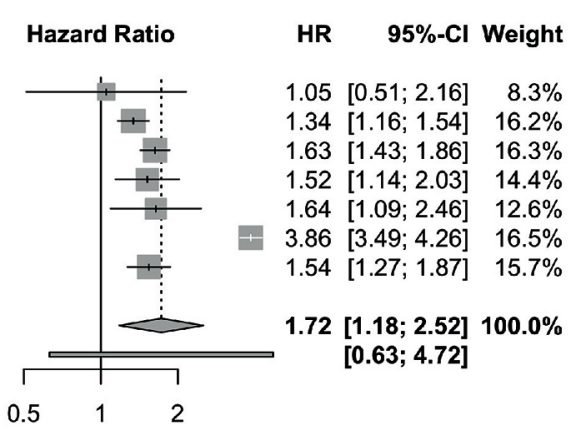

B

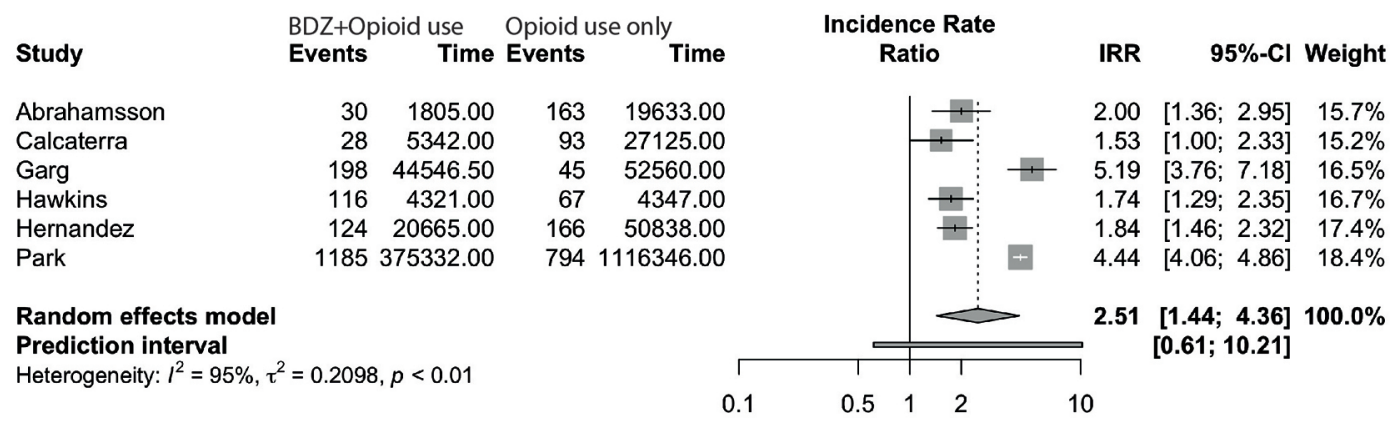

Figure 2 Forrest plots displaying (A) hazard ratio and (B) incidence risk ratio for all available data.

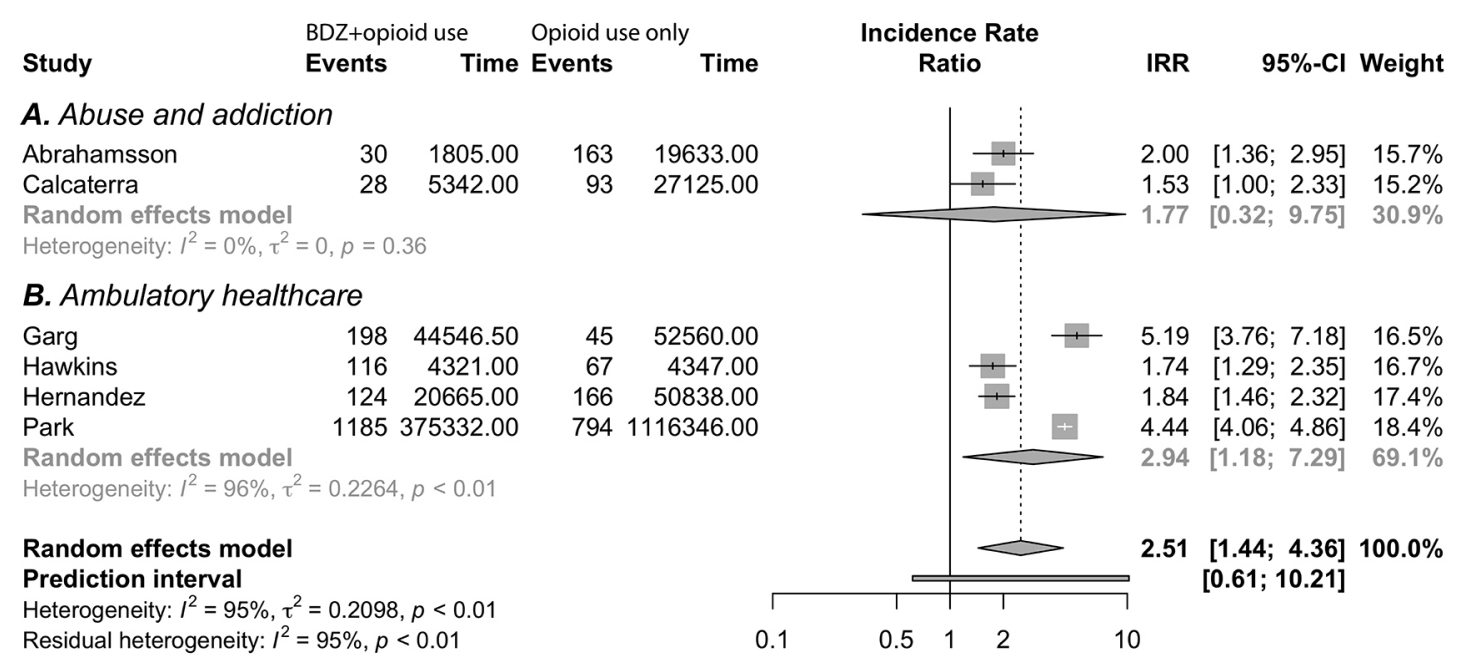

Figure 3 Forrest plot displaying incidence risk ratio for data from (A) abuse and addiction category and (B) ambulatory healthcare.

retrospectively investigated in-hospital cardiopulmonary or respiratory arrest (CPRA) on surgical and medical wards by analysing more than 21 million inpatient discharge records available from Premier database between 2008 and 2013. They found that opioids and sedatives independently and additively increased the risk for CPRA $(26,27)$. Sigurdsson 


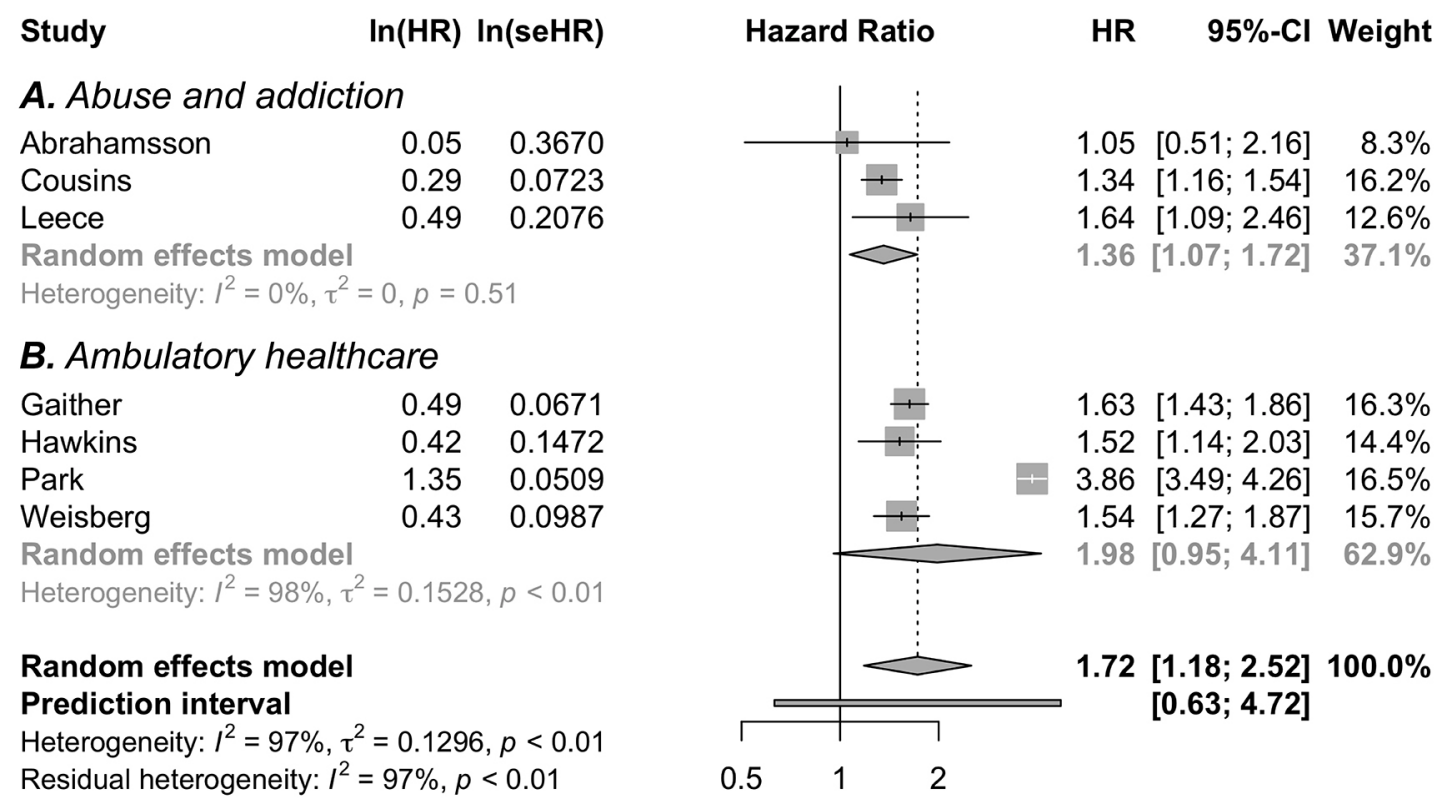

Figure 4 Forrest plot displaying hazard ratio for data from (A) abuse and addiction category and (B) ambulatory healthcare.

et al. investigated the effect of preoperative opioid and/ or benzodiazepine use on postoperative mortality and persistent opioid use after surgery. This study found that single prescriptions for opioids or benzodiazepines filed within 6 months before surgery did not increase the risk for mortality, whereas combined prescription was associated with increased risk for both short-term ( $<30$ days) and longterm mortality (25). An additional finding of this study was that both single and combined preoperative use of opioids and benzodiazepines increased the risk for persistent postoperative opioid use. Unfortunately, the data from the studies in this category were not suitable for pooled analysis.

In conclusion, combined use of opioids and sedatives are likely to increase the risk for in hospital cardiopulmonary and respiratory adverse events and postoperative mortality.

\section{Ambulatory bealthcare}

Twelve studies described various populations on chronic opioid therapy from large US healthcare or insurance databases (28-39). Manuscripts in this section entailed a wide range of subpopulations including patients receiving opioids and benzodiazepines for chronic non cancer pain $(n=7)(29-33,35,37)$, cancer pain $(n=1)(38)$, psychiatric disorders $(n=2)(28,34)$, HIV positive patients $(\mathrm{n}=1)(36)$ and patients with end stage chronic obstructive pulmonary disease ( $n=1)$ (39). Most manuscripts reported about the interaction of opioids with benzodiazepines, but interactions between opioids and gabapentin, alcohol, cocaine and muscle relaxants were also reported. Pooled analyses were performed on suitable data from four studies in this category $(28,29,33,34,36,37,39)$. This showed that the risk for death was higher for combined use of opioids with benzodiazepines [pooled HR 1.98 (0.95-4.11); I I2=98\%; pooled IRR 2.94 (1.18-7.29); I $I^{2}=94 \%$; see part B in Figure 3 and part B in Figure 4]. These results are in line with the overall picture of the data; however, heterogeneity was large and the pooled hazard ratio did not reach statistical significance. In addition, the risk for other serious adverse outcomes was also increased in patients receiving opioids and benzodiazepines.

One study reported on the use of long-term opioid therapy for chronic pain enrolled in the veterans aging cohort study (34). This study found an elevated risk for mortality for co-users, but a protective effect when used in psychotherapeutic interventions. In US veterans with post-traumatic stress disorder, opioids and benzodiazepines elevated the risk of death when compared to single drug and non-users (28). Finally, one manuscript evaluated the use of opioids and benzodiazepines in patients having end stage obstructive lung disease on oxygen therapy (39). Both opioids and benzodiazepines dose dependently increased 
the risk for mortality when used alone or in combination. However, low doses of either agent alone or combined to alleviate dyspnoea appeared safe.

In conclusion, the data in this category suggest that the combined use of opioids and benzodiazepines increases the risk for mortality among a variety of subpopulations.

\section{Discussion}

This manuscript reviewed the effects of concomitant use of opioids and benzodiazepines and found that in most situations this combination results in an elevated risk of death or serious harm. Most manuscripts reported on the drug interaction in patients of the abuse and addiction and the ambulatory healthcare settings. For these settings, we were able to conduct pooled analyses of suitable data from 10 studies to provide a quantification of the increased risk for mortality $(11,12,18,22,28,29,33,34,36,37)$. These analyses indicated a significant effect of combined opioid benzodiazepine use on mortality. However, in terminal patients no negative effects of the combination were found (24). Before we separately discuss each category, we will first discuss the main actions of opioids and benzodiazepines on the central nervous system.

Opioids are derivatives of the naturally occurring opium peptide morphine that act on opioid receptors at cellular level. The most prominent of these receptors, the $\mu$-opioid receptor, is associated with the analgesic, respiratory depressant and rewarding effects of opioids. Benzodiazepines entail a class of sedative drugs that share the same core chemical structure of a fused benzene and diazepine ring. Benzodiazepines are allosteric agonists of the GABAa receptor and predominantly bind the a1 and a2 subunits of this receptor, inhibiting neuronal signal transmission. The affinity of the various types of benzodiazepines to the alpha units on the GABAa receptor determines their predominant clinical effect (i.e., sedation or anxiolysis). Apart from their beneficial effects, both opioids and benzodiazepines negatively interfere with respiratory control. The drive to breathe is controlled by neurons of the respiratory centres in the brainstem. Since both opioid and GABAa receptors are expressed on respiratory neurons, opioids and benzodiazepines profoundly disrupt physiologic control of breathing. Opioids' main effect is a reduction in respiratory rate which is caused by its direct inhibitory effects on mu receptors in the brainstem (40). In contrast, benzodiazepine respiratory depression is primarily characterized by a reduction in tidal volume. This is demonstrated by a decreased slope of the ventilatory response curve to carbon dioxide rather than the rightward shift of the curve that is seen with opioids $(41,42)$. In addition to these effects on respiratory drive, both benzodiazepines and opioids reduce upper airway patency and cause obstructive apneas and hypopneas (43). A pharmacokinetic interaction between these agents may also play a role in their effect on respiration. For instance, some opioids are metabolized via CYP3A4, an enzyme that is inhibited by alprazolam. Combined use of these agents would result in elevated opioid serum concentrations causing a prolonged effect.

Thus, it is clear that opioids and benzodiazepines both negatively interfere with respiration. Indeed, both animal and human studies have shown that the combination of opioids with benzodiazepines lead to more hypoxia and hypercapnia when compared with single use of these agents (44-47). Since we anticipated that risk and benefits of combined opioid-sedative use would be dependent on the clinical use case, eligible manuscripts were categorized as follows: abuse and addition, palliative care, inpatient and outpatient settings.

\section{Abuse and addition}

The literature suggests that polypharmacy with benzodiazepines, cocaine, antidepressants, pregabalin and alcohol is commonly encountered when analyzing lethal overdoses by patients abusing opioids or on opioid replacement therapy. The data unequivocally suggests combining these drugs with opioids increases the risk for fatal overdose. There may be various reasons why drug abusers use a combination of drugs. Many drug abusers suffer from concomitant psychiatric disorders, rendering them at risk for (poly-)drug abuse in the first place. Second, benzodiazepines amplify the pleasurable effects of opioids (48). For instance, heroin users report a more intense and prolonged effect when concomitantly using a benzodiazepine. These effects are known to heroin users, but also noted by subjects on methadone or buprenorphine replacement therapy, who report a "heroin-like" profound drug effect when these agents are concomitantly used with a benzodiazepine $(49,50)$. Nevertheless, subjects on opioid replacement therapy are often co-prescribed benzodiazepines to treat underlying psychiatric disorders and to facilitate treatment compliance. Part of the hazard of combined use is that the tolerance to respiratory depression by chronic opioid users does not imply tolerance 
to respiratory depression for benzodiazepines. This causes a high risk of overdose even among experienced opioid abusers (6). In addition, clinicians may erroneously expect their patients on opioid replacement therapy to require larger doses of sedatives to obtain an adequate treatment effect.

The combination of benzodiazepines with buprenorphine or methadone deserves special attention. Although buprenorphine is known for its ceiling effect on respiratory depression, this effect is only observed without the concurrent use of other central nervous system depressing agents (51). Indeed, post-mortem data from Hakkinen et al. show that benzodiazepines and alcohol were found in 82 and $58 \%$ of deceased subjects using buprenorphine (21). Nevertheless, there are indications that the risk for lethal adverse events caused by concomitant (mis-)use is lower with buprenorphine compared to methadone $(12,19)$. This observation warrants further research as the choice between buprenorphine and methadone may have significant consequences for the risk of adverse events.

\section{Palliative healthcare}

One manuscript reported on the effect of the combined use of opioids, benzodiazepines and antipsychotics in the hospice setting (24). In this study, the combined use of opioids with benzodiazepines and antipsychotics, appeared not to negatively affect survival time or increase the risk of night-time death. It is known that opioids and benzodiazepines alone do not significantly compromise respiratory function or affect survival time in terminally ill patients (52-54). This is consistent with Clemens et al., who showed that in terminally ill patients suffering from anxiety and dyspnoea the combination of lorazepam and morphine was both effective and safe. Mean oxygen saturation did not change significantly after initiation of therapy, whereas dyspnoea and respiratory rate diminished notably (55). Although these investigators did not study survival length, data from our review support these findings and indicate that the combined use of these agents may be safe in this population.

Counterintuitively, the data from Golčić et al. also show that patients on high dose opioid therapy survived longer compared to patients receiving lower doses (24). A caveat here is that patients receiving more than 300 oral morphine equivalents (OME) per day were on average younger. Nevertheless, the trend points in the same direction when looking at all dose ranges; even in the lower dose ranges, in which age did not differ so much, a dose dependent decrease in the hazard ratio was observed, albeit not statistically significant. We speculate that high pain levels that are often encountered in terminally ill patients warrant higher opioid doses to achieve optimal nociception-anti nociception (i.e., pain - analgesic) balance.

\section{Inpatient healthcare}

The data from our review show that concomitant opioid benzodiazepine use in hospitalized patients increase the risk for adverse events on both surgical and medical wards (25-27). These results add to the results of a recent meta-analysis that investigated risk factors associated with opioid induced respiratory depression (OIRD) after surgery (56). It was found that concurrent prescription of sedatives was present in $56 \%$ of patients with OIRD. In addition, it appeared that OIRD occurred most frequently in the first 24 hours after surgery and that patients with coexisting cardiac disease, pulmonary disease and sleep disordered breathing were at highest risk (56). These findings largely correspond with the data from this review. Evidently, recognition of patients who are at risk for respiratory complications is essential to optimize postoperative care in order to minimize adverse events. The results of this review and the meta-analysis suggest that opioids and sedatives should be avoided in high risk patients whenever possible. If not possible to avoid, these patients should have their respiration monitored electronically and continuously for early detection of OIRD.

\section{Ambulatory bealthcare settings}

The data from this group entailed the biggest proportion of studies included in this review. On average, these were large database studies conducted in various subpopulations, which shared the setting of representing out-of-hospital or ambulatory healthcare (28-39). The data uniformly indicated that combined use of opioids and sedatives (mostly benzodiazepines, but also gabapentin, alcohol, muscle relaxants, cocaine, etc.) increased the risk for mortality among all subpopulations. Exposure-response evaluations showed that the risk for mortality was highest in patients that had recently commenced treatment (29) or had a history of benzodiazepine use (37). These risk factors add to other known risk factors for adverse outcomes such as older age, high opioid daily dose, and long duration of opioid use (57). In addition, two manuscripts in this review evaluated 
the effect of co-prescription of opioids and benzodiazepines to patients with post-traumatic stress disorder or substance abuse disorders $(28,34)$. Concurrent use of opioids with benzodiazepines was associated with increased risk for mortality, whereas psychological interventions aimed to treat underlying psychiatric illness appeared to be protective. Minimizing concurrency and close follow up with psychological interventions should therefore be considered in these patients. Finally, Ekström et al. evaluated concurrent use of opioids and benzodiazepines in patients with severe obstructive lung disease (39). They found that benzodiazepines and opioids dose dependently increased mortality, although low doses appeared to be safe and did not increase the risk for mortality during hospital admission. These results are in line with previous data showing that low dose opioids or benzodiazepines do not increase the risk for severe adverse events or significantly alter blood gas results in these patients (58-60).

Our review has several limitations. First, although we identified a considerable number of manuscripts, we only searched the PubMed database for manuscripts written in English language and, as such, we may have missed valuable manuscripts. Most data came from retrospective and post-mortem studies whose results may be confounded by unknown variables. We performed analyses of pooled data when available and separately for hazard ratios and incidence risk ratios. Since only ten studies could be included in these analyses and because of the high degree of heterogeneity, the results should be regarded as indicative rather than absolute measures of the risk for mortality.

We focused on interactions between opioids and benzodiazepines and did not give extensive attention to other CNS active agents, such as alcohol and antidepressants. Thus, our conclusions for interactions between opioids and sedatives other than benzodiazepines are exploratory.

Lastly, this review focused on the impact of opioids and benzodiazepines on patient harm (i.e., mortality and severe respiratory adverse events) and we did not specifically review the benefits of the drug combination. This is important, as the chance of benefit versus the chance of harm [i.e., risk/benefit ratio or the utility, see review of Van Dam et al. (61)] is always a consideration when prescribing a drug or drug combination for a patient. Our conclusion is that the utility of the opioid-benzodiazepine combination is negative (i.e., the chance of harm is greater than the chance of benefit) for the majority of clinical uses. However, utility critically depends on the clinical context and the dose and type of opioid and sedative and we identified a possible positive utility in patients suffering from terminal illness.

In conclusion, this review indicates that the combined use of opioids with benzodiazepines increases the risk for severe adverse respiratory events and the risk for mortality over a broad line of clinical and non-clinical settings. However, there are situations, such as in the hospice setting, where benefits may outweigh the risks.

\section{Acknowledgments}

Funding: None.

\section{Footnote}

Provenance and Peer Review: This article was commissioned by the Guest Editor (Mellar P. Davis) for the series "Opioid Utility the Other Half of Equianalgesia" published in Annals of Palliative Medicine. The article was sent for external peer review organized by the Guest Editor and the editorial office.

Conflicts of Interest: The series "Opioid Utility the Other Half of Equianalgesia" was commissioned by the editorial office without any funding or sponsorship. The authors have no other conflicts of interest to declare.

Ethical Statement: The authors are accountable for all aspects of the work in ensuring that questions related to the accuracy or integrity of any part of the work are appropriately investigated and resolved.

Open Access Statement: This is an Open Access article distributed in accordance with the Creative Commons Attribution-NonCommercial-NoDerivs 4.0 International License (CC BY-NC-ND 4.0), which permits the noncommercial replication and distribution of the article with the strict proviso that no changes or edits are made and the original work is properly cited (including links to both the formal publication through the relevant DOI and the license). See: https://creativecommons.org/licenses/by-nc-nd/4.0/.

\section{References}

1. Bedene A, Lijfering WM, Niesters $M$, et al. Opioid Prescription Patterns and Risk Factors Associated With Opioid Use in the Netherlands. JAMA Netw Open 2019;2:e1910223. 
2. Centers for Disease Control and Prevention 2018 Annual surveillance report of drug-related risks and outcomes. Available online: https://www.cdc.gov/drugoverdose/pdf/ pubs/2018-cdc-drug-surveillance-report.pdf. Accessed November 20, 2019.

3. Agarwal SD, Landon BE. Patterns in Outpatient Benzodiazepine Prescribing in the United States. JAMA Netw Open 2019;2:e187399.

4. Kurko TA, Saastamoinen LK, Tahkapaa S, et al. Longterm use of benzodiazepines: Definitions, prevalence and usage patterns - a systematic review of register-based studies. Eur Psychiatry 2015;30:1037-47.

5. Kan CC, Hilberink SR, Breteler MH. Determination of the main risk factors for benzodiazepine dependence using a multivariate and multidimensional approach. Compr Psychiatry 2004;45:88-94.

6. White JM, Irvine RJ. Mechanisms of fatal opioid overdose. Addiction 1999;94:961-72.

7. Gladden RM, O'Donnell J, Mattson CL, et al. Changes in Opioid-Involved Overdose Deaths by Opioid Type and Presence of Benzodiazepines, Cocaine, and Methamphetamine - 25 States, July-December 2017 to January-June 2018. MMWR Morb Mortal Wkly Rep 2019;68:737-44.

8. Dowell D, Haegerich TM, Chou R. CDC Guideline for Prescribing Opioids for Chronic Pain--United States, 2016. JAMA 2016;315:1624-45.

9. R Core Team. R: a language and environment for statistical computing. R Foundation for Statistical Computing, Vienna, Austria. 2018. Available online: https://www. R-project.org/

10. Viechtbauer $W$. Conducting meta-analyses in $\mathrm{R}$ with the metafor package. J Statist Softw 2010:1-48. Available online: https://www.jstatsoft.org/article/view/

11. Calcaterra SL, Severtson SG, Bau GE, et al. Trends in intentional abuse or misuse of benzodiazepines and opioid analgesics and the associated mortality reported to poison centers across the United States from 2000 to 2014. Clin Toxicol (Phila) 2018;56:1107-14.

12. Abrahamsson T, Berge J, Ojehagen A, et al. Benzodiazepine, z-drug and pregabalin prescriptions and mortality among patients in opioid maintenance treatment-A nation-wide register-based open cohort study. Drug Alcohol Depend 2017;174:58-64.

13. Pfister GJ, Burkes RM, Guinn B, et al. Opioid overdose leading to intensive care unit admission: Epidemiology and outcomes. J Crit Care 2016;35:29-32.

14. Petrushevska T, Jakovski Z, Poposka V, et al. Drug-related deaths between 2002 and 2013 with accent to methadone and benzodiazepines. J Forensic Leg Med 2015;31:12-8.

15. Visconti AJ, Santos GM, Lemos NP, et al. Opioid Overdose Deaths in the City and County of San Francisco: Prevalence, Distribution, and Disparities. J Urban Health 2015;92:758-72.

16. Pavarin RM. Mortality Risk Among Heroin Abusers: Clients and Non-clients of Public Treatment Centers for Drug Addiction. Subst Use Misuse 2015;50:1690-6.

17. Nielsen MK, Johansen SS, Linnet K. Evaluation of polydrug use in methadone-related fatalities using segmental hair analysis. Forensic Sci Int 2015;248:134-9.

18. Leece P, Cavacuiti C, Macdonald EM, et al. Predictors of Opioid-Related Death During Methadone Therapy. J Subst Abuse Treat 2015;57:30-5.

19. Lee SC, Klein-Schwartz W, Doyon S, et al. Comparison of toxicity associated with nonmedical use of benzodiazepines with buprenorphine or methadone. Drug Alcohol Depend 2014;138:118-23.

20. Calcaterra S, Glanz J, Binswanger IA. National trends in pharmaceutical opioid related overdose deaths compared to other substance related overdose deaths: 1999-2009. Drug Alcohol Depend 2013;131:263-70.

21. Häkkinen M, Launiainen T, Vuori E, et al. Benzodiazepines and alcohol are associated with cases of fatal buprenorphine poisoning. Eur J Clin Pharmacol 2012;68:301-9.

22. Cousins G, Teljeur C, Motterlini N, et al. Risk of drugrelated mortality during periods of transition in methadone maintenance treatment: a cohort study. J Subst Abuse Treat 2011;41:252-60.

23. Mirakbari SM, Innes GD, Christenson J, et al. Do cointoxicants increase adverse event rates in the first 24 hours in patients resuscitated from acute opioid overdose? J Toxicol Clin Toxicol 2003;41:947-53.

24. Golčić M, Dobrila-Dintinjana R, Golcic G, et al. The Impact of Combined Use of Opioids, Antipsychotics, and Anxiolytics on Survival in the Hospice Setting. J Pain Symptom Manage 2018;55:22-30.

25. Sigurdsson MI, Helgadottir S, Long TE, et al. Association Between Preoperative Opioid and Benzodiazepine Prescription Patterns and Mortality After Noncardiac Surgery. JAMA Surg 2019. [Epub ahead of print].

26. Izrailtyan I, Qiu J, Overdyk FJ, et al. Risk factors for cardiopulmonary and respiratory arrest in medical and surgical hospital patients on opioid analgesics and sedatives. PLoS One 2018;13:e0194553.

27. Overdyk FJ, Dowling O, Marino J, et al. Association of 
Opioids and Sedatives with Increased Risk of In-Hospital Cardiopulmonary Arrest from an Administrative Database. PLoS One 2016;11:e0150214.

28. Hawkins EJ, Goldberg SB, Malte CA, et al. New Coprescription of Opioids and Benzodiazepines and Mortality Among Veterans Affairs Patients With Posttraumatic Stress Disorder. J Clin Psychiatry 2019. doi: 10.4088/JCP.18m12689.

29. Hernandez I, He M, Brooks MM, et al. ExposureResponse Association Between Concurrent Opioid and Benzodiazepine Use and Risk of Opioid-Related Overdose in Medicare Part D Beneficiaries. JAMA Netw Open 2018;1:e180919.

30. Gressler LE, Martin BC, Hudson TJ, et al. Relationship between concomitant benzodiazepine-opioid use and adverse outcomes among US veterans. Pain 2018;159:451-9.

31. Sun EC, Dixit A, Humphreys K, et al. Association between concurrent use of prescription opioids and benzodiazepines and overdose: retrospective analysis. BMJ 2017;356:j760.

32. Gomes T, Juurlink DN, Antoniou T, et al. Gabapentin, opioids, and the risk of opioid-related death: A population-based nested case-control study. PLoS Med 2017;14:e1002396.

33. Garg RK, Fulton-Kehoe D, Franklin GM. Patterns of Opioid Use and Risk of Opioid Overdose Death Among Medicaid Patients. Med Care 2017;55:661-8.

34. Gaither JR, Goulet JL, Becker WC, et al. The Effect of Substance Use Disorders on the Association Between Guideline-concordant Long-term Opioid Therapy and All-cause Mortality. J Addict Med 2016;10:418-28.

35. Dasgupta N, Funk MJ, Proescholdbell S, et al. Cohort Study of the Impact of High-Dose Opioid Analgesics on Overdose Mortality. Pain Med 2016;17:85-98.

36. Weisberg DF, Gordon KS, Barry DT, et al. Long-term Prescription of Opioids and/or Benzodiazepines and Mortality Among HIV-Infected and Uninfected Patients. J Acquir Immune Defic Syndr 2015;69:223-33.

37. Park TW, Saitz R, Ganoczy D, et al. Benzodiazepine prescribing patterns and deaths from drug overdose among US veterans receiving opioid analgesics: case-cohort study. Bmj 2015;350:h2698.

38. Chang WP, Lin CC. Use of opioid analgesics or sleeping medication and survival of cancer patients. Eur J Oncol Nurs 2015;19:199-206.

39. Ekström MP, Bornefalk-Hermansson A, Abernethy AP, et al. Safety of benzodiazepines and opioids in very severe respiratory disease: national prospective study. BMJ 2014;348:g445.

40. Smith JC, Ellenberger HH, Ballanyi K, et al. PreBotzinger complex: a brainstem region that may generate respiratory rhythm in mammals. Science 1991;254:726-9.

41. Forster A, Gardaz JP, Suter PM, et al. Respiratory depression by midazolam and diazepam. Anesthesiology 1980;53:494-7.

42. Gross JB, Smith L, Smith TC. Time course of ventilatory response to carbon dioxide after intravenous diazepam. Anesthesiology 1982;57:18-21.

43. Bernards CM, Knowlton SL, Schmidt DF, et al. Respiratory and sleep effects of remifentanil in volunteers with moderate obstructive sleep apnea. Anesthesiology 2009;110:41-9.

44. Afzal A, Kiyatkin EA. Interactions of benzodiazepines with heroin: Respiratory depression, temperature effects, and behavior. Neuropharmacology 2019;158:107677.

45. Bailey PL, Pace NL, Ashburn MA, et al. Frequent hypoxemia and apnea after sedation with midazolam and fentanyl. Anesthesiology 1990;73:826-30.

46. Gueye PN, Borron SW, Risede P, et al. Buprenorphine and midazolam act in combination to depress respiration in rats. Toxicol Sci 2002;65:107-14.

47. Lintzeris N, Mitchell TB, Bond AJ, et al. Pharmacodynamics of diazepam co-administered with methadone or buprenorphine under high dose conditions in opioid dependent patients. Drug Alcohol Depend 2007;91:187-94.

48. Walker BM, Ettenberg A. Benzodiazepine modulation of opiate reward. Exp Clin Psychopharmacol 2001;9:191-7.

49. Stitzer ML, Griffiths RR, McLellan AT, et al. Diazepam use among methadone maintenance patients: patterns and dosages. Drug Alcohol Depend 1981;8:189-99.

50. Chen KW, Berger CC, Forde DP, et al. Benzodiazepine use and misuse among patients in a methadone program. BMC Psychiatry 2011;11:90.

51. Faroqui MH, Cole M, Curran J. Buprenorphine, benzodiazepines and respiratory depression. Anaesthesia 1983;38:1002-3.

52. López-Saca JM, Centeno C. Opioids prescription for symptoms relief and the impact on respiratory function: updated evidence. Curr Opin Support Palliat Care 2014;8:383-90.

53. Simon ST, Higginson IJ, Booth S, et al. Benzodiazepines for the relief of breathlessness in advanced malignant and non-malignant diseases in adults. Cochrane Database Syst Rev 2016;10:CD007354.

54. Good PD, Ravenscroft PJ, Cavenagh J. Effects of opioids 
and sedatives on survival in an Australian inpatient palliative care population. Intern Med J 2005;35:512-7.

55. Sykes AJ. Budget holding. Br J Gen Pract 1990;40:170.

56. Gupta K, Nagappa M, Prasad A, et al. Risk factors for opioid-induced respiratory depression in surgical patients: a systematic review and meta-analyses. BMJ Open 2018;8:e024086.

57. Sharma V, Weir D, Samanani S, et al. Characterisation of concurrent use of prescription opioids and benzodiazepine/ Z-drugs in Alberta, Canada: a population-based study. BMJ Open 2019;9:e030858.

58. Simon ST, Higginson IJ, Booth S, et al. Benzodiazepines for the relief of breathlessness in advanced malignant and

Cite this article as: Boon M, van Dorp E, Broens S, Overdyk F. Combining opioids and benzodiazepines: effects on mortality and severe adverse respiratory events. Ann Palliat Med 2020;9(2):542-557. doi: 10.21037/apm.2019.12.09 non-malignant diseases in adults. Cochrane Database Syst Rev 2010;(1):CD007354.

59. Jennings AL, Davies AN, Higgins JP, et al. A systematic review of the use of opioids in the management of dyspnoea. Thorax 2002;57:939-44.

60. Stege G, Heijdra YF, van den Elshout FJ, et al. Temazepam $10 \mathrm{mg}$ does not affect breathing and gas exchange in patients with severe normocapnic COPD. Respir Med 2010;104:518-24.

61. van Dam CJ, Algera H, Olofsen E, et al. Opioid utility function: methods and implications. Ann Palliat Med 2019. DOI: 10.21037/apm.2019.10.09. 